\section{Application of Different methods for Reducing Radiation Dose to Breast during MDCT}

\author{
Keshtkar M. ${ }^{1}$, Saba V. ${ }^{1 *}$, Mosleh-Shirazi M. A. ${ }^{2}$
}

\begin{abstract}
The increased use of computed tomography (CT) and its high radiation dose have led to great concerns about its potential for radiation induced cancer risks. Breast is a radiosensitive tissue based on tissue weighting factors assigned by the International Commission on Radiological Protection (ICRP). Moreover, the dose is maximal on the surface of the patient. Therefore, strategies should be taken to reduce radiation dose to the breast. The aim of this review is to introduce methods used for reducing radiation dose to breast in thoracic $\mathrm{CT}$ and review related performed studies. The literature indicates that bismuth shielding increases image noise and CT numbers as well as introducing streak artifacts. Tube current modulation (TCM) technique and iterative reconstruction algorithms can provide some levels of dose reduction to radiosensitive organs and superior image quality without the disadvantages of bismuth shielding. However, they are not available on all CT scanners, especially in low-income countries. Such centers may have to continue using bismuth shields to reduce the dose until these superior techniques become available at lower costs in all CT scanners. Furthermore, design and manufacture of new shields with the lower impact on image quality are desirable.
\end{abstract}

\section{Keywords}

Breast, Dose Reduction, Computed Tomography, Bismuth Shielding

\section{Introduction}

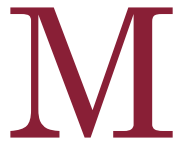

ultidetector computed tomography (MDCT) as an advanced technology plays an important role in medical diagnosis. The use of computed tomography (CT) has increased remarkably since its advent. Based on a report in United States, the number of CT examinations performed in 1980 was 3 million, whereas, it increased to more than 68.7 million in 2007 [1, 2]. This increased use of CT elevated predicted cancer risks attributable to CT from $0.4 \%$ to $1.5-2.0 \%$ [1].

Although CT accounts for $11 \%$ of all X-ray based procedures, contributes more than $70 \%$ of the total medically related radiation exposures to patients. The increased use of $\mathrm{CT}$ and its high radiation doses have led to great concerns about its potential for radiation induced cancer risks. This is more critical for children, due to their higher sensitivity to radiation [3].

The biological effects of radiation exposure are classified in two groups: stochastic effects and deterministic effects. Deterministic effects do not occur below the threshold dose. Stochastic effects occur by chance and may cause cancer or genetic effects in irradiated individuals
${ }^{1}$ Radiology Department, Faculty of Paramedicine, Aja University of Medical Sciences, Tehran, Iran

${ }^{2}$ Ionizing and Non-Ionizing Radiation Protection

Research Center and

Department of Radio-

Oncology, Shiraz Univer-

sity of Medical Sciences,

Shiraz

*Corresponding author: V. Saba

Radiology Department,

Faculty of Paramedicine

Aja University of Medical

Sciences, Etemadzadeh

Ave, Fatemi St, Tehran,

Iran

E-mail: vsaba@aut.ac.ir

Received: 23 July 2018

Accepted: 7 August 2018 
or their offspring. The radiation dose levels related to CT examinations are mainly attributed to stochastic effects. But, it can be reached to threshold doses in the case of repeated exposure to ionizing radiation [4].

Breast is a radiosensitive tissue based on tissue weighting factors assigned by the International Commission on Radiological Protection (ICRP) [5]. Moreover, the dose is maximal on the surface of the patient [6-8]. Incidence of breast cancer has increased in populations who received doses similar to CT scans [4]. It should be noted that breast cancer is prevalent and is the second leading cause of cancer related deaths among women [9-13]. Therefore, strategies must be used in order to reduce radiation dose to these organs. The aim of this study is to introduce methods used to reduce radiation dose to breast in thoracic $\mathrm{CT}$ and review related performed studies.

Dose reduction in CT may be done by scanning parameters modification and special techniques. Scanning parameters can be controlled by technologist and include scanner geometry, tube current and voltage, scanning modes, length, collimation, table speed and pitch, and gantry rotation time [2]. Scanning parameters modification are not discussed in this paper, and only special techniques are introduced. Special techniques are consist of organ-based tube current modulation (TCM), globally tube current reduction, reconstruction algorithms and shielding [14].

Effectiveness of dose reduction techniques should be assessed by the amount of reduction in dose and their impact on image quality. Dosimetric assessment may be done by patient study or using phantoms especially inhomogeneous anthropomorphic phantoms [15]. Qualitative and quantitative assessments can be considered for an image quality evaluation. Quantitative assessment may be performed in regards to CT number accuracy and image noise. CT numbers and mage noise are measured as mean of Hounsfield Unit (HU) and standard deviation of HU in the region of in- terests, respectively.

\section{Organ-based TCM}

In this technique, the tube current for the approximately $120^{\circ}$ anterior arc of the X-ray source's rotation is reduced by approximately $75 \%$. During the remaining arc of the angular rotation, the tube current increased to compensate for the reduced anterior current [16].

There are several studies that evaluated the effectiveness of organ-based TCM for breast dose saved during CT. Coursey et al. (2008) reported that for pediatric chest MDCT and automatic tube current modulation when the shield was placed after the scout and the shield was placed before the scout, reduced breast dose by $35 \%$ and $20 \%$, respectively. Automatic tube current modulation when the shield was placed after the scout and when the shield was placed before the scout increased image noise in the superior mediastinum by $4.2 \mathrm{HU}$ and 1.9 HU, respectively [17].

Jia Wang et al. (2011) reported that organbased TCM reduced the breast dose by $34-$ $39 \%$ depending on phantom sizes. Increased noise with organ-based TCM was insignificant and streak artifact was not observed [18]. A patient study by Kim et al. (2013) reported dose reduction of $20.8 \%$ to the superficial breast tissue when organ-based TCM was used. [19].

One limitation of organ-based TCM is that this technique increased dose to posteriorly and laterally located structures. A study by Hoang et al. reported that organ-based TCM increased dose to upper lungs and spinal bone marrow by $29 \%$ and $15-20 \%$, respectively [20]. A recently introduced technique named as Organ Dose Modulation reduces tube current anteriorly while maintaining baseline tube current level at the rest of angular rotation [16]. Lambert and Gould (2016) reported $38 \%$ dose saving due to Organ Dose Modulation. Organ Dose Modulation increased anterior image noise by $0.8 \mathrm{HU}$. The mean CT numbers in the chest region changed by -0.2 $\mathrm{HU}$ in the Organ Dose Modulation mode [16]. 
Another limitation of organ-based TCM exists for patients with larger breasts in which a portion of the breast tissue may position laterally, hence, receiving high radiation doses [19]. A study reported 50\% increase to breast dose due to off-center positioning of phantom while using organ-based TCM [21].

\section{Global reduction of tube current}

In this technique, tube current globally (i.e., over all $360^{\circ}$ ) decreased [18]. The most practical way of deceasing CT radiation dose is a reduction in tube current [2]. However, tube current reduction may affect diagnostic quality of CT image by increasing image noise [2, 18]. The majority of publications proved that global tube current reduction has no impacts on CT number accuracy. Jia Wang (2011) reported $37 \%$ breast dose reduction due to global tube current reduction, while image noise increased. Additionally, streak and beam hardening artifacts were not observed [18]. Foley et al. (2013) reported that global tube current reduction reduced dose to the breast $20 \%$. Global tube current reduction had no impacts on CT numbers, only increased image noise. [22].

\section{Iterative reconstruction}

Filtered back projection techniques are currently used for CT reconstruction algorithm that does not produce acceptable diagnostic images if the tube current is markedly reduced. Newer reconstruction algorithms are iterative reconstructions allowing high quality image acquisition at a lower tube current, while reducing image noise [14]. Adaptive statistical iterative reconstruction is one example that has been studied recently as a dose reduction method. Mathieu and Cody reported 30\% to $44 \%$ dose reduction due to using Adaptive statistical iterative reconstruction on a phantom during a routine chest CT [23].

\section{Bismuth shielding}

Another dose reduction technique is the use of bismuth shields that are cheap and easy to use. Bismuth shields are different from lead shields in which all x-ray radiation is blocked. Bismuth shields protect superficial radiosensitive organs such as eyes, thyroid and breast by attenuating primary $\mathrm{x}$-ray beams. Using bismuth for dose saving in CT scan has two advantages over lead; first, bismuth provides greater attenuation than lead, especially at higher tube potential settings used in CT. Second, bismuth products are more flexible and can be mold to the body's surface easily [24].

The majority of studies proved remarkable breast dose reduction efficacies of bismuth shielding $[16,25,26]$. A patient study by Hopper (2002), showed that 1 thickness of bismuth shield $\left(0.85 \mathrm{gr} / \mathrm{cm}^{2}\right.$ bismuth) saved absorbed dose to the breast approximately $52.4 \%$ [24].

Hohl et al. (2006) reported that bismuth shielding resulted in $47 \%$ organ dose reduction for the breast [27]. Catuzzo et al. (2010) assessed the bismuth breast shield by in vivo and phantom measurements. Organ dose evaluation in the anthropomorphic phantom showed $59 \%$ dose reduction due to bismuth breast shielding. In vivo measurements showed that bismuth shield reduced surface breast dose by $41 \%$. [28].

Einstein et al. (2011) reported that bismuth shielding decreased breast dose during coronary CT angiography by $46 \%-57 \%$ depending on breast size and scanning protocol [29]. Jia Wang (2011) reported that bismuth shielding reduced breast dose by $37 \%$ [18].

The most publications reported three effects of bismuth shields on the image quality. First, increasing image noise; Hohl et al. (2006) showed that image noise in central breast tissue without shielding was $6.9 \mathrm{HU}$ and it increased to $10.1 \mathrm{HU}$ due to bismuth shielding. When the $1 \mathrm{~cm}$ spacer was used between a shield and skin, noise increased to 9.8 HU [27]. Einstein et al. (2011) reported that shielding decreased contrast to noise ratio by $20.9 \%$ and increased image noise significantly that may affect coronary artery visualization. Jia Wang (2011) re- 
ported that bismuth shielding increased image noise [18].

Second, increasing CT number; Tappouni and Mathers (2012) revealed that bismuth shielding increased CT number of anterior chest region by $20 \mathrm{HU}$ [26]. Lambert and Gould (2016) reported that bismuth shielding increased the mean $\mathrm{CT}$ numbers in the chest region by $12.4 \mathrm{HU}$ [16].

Third, causing artifacts; Jia Wang (2011) reported that bismuth shielding introduced streak and beam hardening artifacts [18].

\section{Conclusion}

It can be seen that dose reduction techniques reduce breast dose $26-59 \%$, depending on different sizes of phantoms and different protocols. However, a crucial issue in using dose reduction techniques is their impact on the image quality. The literature indicates that bismuth shielding increases image noise and CT numbers as well as introducing streak artifacts. Obviously if the degree of the resulting changes on the image quality is still diagnostically acceptable, the use of such shielding causes no serious concern. Organ-based TCM, iterative reconstruction algorithms and global tube current reduction do not significantly change CT numbers and cause artifacts. Organ-based TCM increases image noise insignificantly, however, global tube current reduction increases image noise similar to bismuth shielding. These findings have led to the American association of Medical Physicists (AAPM) stating that TCM and iterative reconstruction algorithms can provide some levels of dose reduction to radiosensitive organs at the superior image quality without the disadvantages of bismuth shielding. However, they are not available on all CT scanners, especially in low-income countries. The scanners in such centers may not be equipped with TCM and iterative reconstruction algorithms for years. Such centers may continue using bismuth shields to reduce doses until these superior techniques become available at lower costs in all CT scanners. While using bismuth shields, two points must be considered: firstly, placing a spacer between the shield and skin (to decrease the potential of artifacts), and then, the shield should be placed on the patient after obtaining the scout image (to avoid tube current compensation). Furthermore, design and manufacture of new shields with the lower impact on image quality is desirable.

\section{Conflict of Interest \\ None}

\section{References}

1. Brenner DJ, Hall EJ. Computed tomography--an increasing source of radiation exposure. $N$ Engl J Med. 2007;357:2277-84. doi: 10.1056/NEJMra072149. PubMed PMID: 18046031.

2. Kalra MK, Maher MM, Toth TL, Hamberg LM, Blake MA, Shepard JA, et al. Strategies for CT radiation dose optimization. Radiology. 2004;230:619-28. doi: 10.1148/radiol.2303021726. PubMed PMID: 14739312.

3. Karami V, Zabihzadeh M, Keshtkar M. Evaluation of the Entrance Surface Dose (ESD) and Radiation Dose to the Radiosensitive Organs in Pediatric Pelvic Radiography. Int J Pediatr. 2017;5:5013-22.

4. Curtis JR. Computed tomography shielding methods: a literature review. Radiol Technol. 2010;81:428-36. PubMed PMID: 20445137.

5. Wrixon AD. New ICRP recommendations. J Radiol Prot. 2008;28:161-8. doi: 10.1088/09524746/28/2/R02. PubMed PMID: 18495983.

6. Zakariaee SS, Saba V, Valizadeh A. Study the Effect of Gantry Tilting and Tube Voltage Reducing on the Eye Lens Dose Reduction in Computed Tomography Using MCNPx. Paramedical Sciences and Military Health. 2017;12:39-49.

7. Zeinali-Rafsanjani $B$, Faghihi $R$, Mosleh-Shirazi MA, Mosalaei A, Hadad K. Revision of orthovoltage chest wall treatment using Monte Carlo simulations. Technol Health Care. 2017;25:413-24. doi: 10.3233/THC-161276. PubMed PMID: 27886021.

8. Mohammadyari P, Faghihi R, Mosleh-Shirazi MA, Lotfi M, Hematiyan MR, Koontz C, et al. Calculation of dose distribution in compressible breast tissues using finite element modeling, Monte Carlo simulation and thermoluminescence dosimeters. Phys Med Biol. 2015;60:9185-202. doi: 10.1088/00319155/60/23/9185. PubMed PMID: 26572554.

9. Zeinali Rafsanjani B, Mosleh-Shirazi MA, Faghihi 
R, Mosalaei A, Omidvari S, Hadad K, et al. Breast cancer and its radiotherapeutic methods. Iranian Journal of Medical Physics. 2012;9:75-85.

10. Keshtkar M, Shahbazi-Gahrouei D, Khoshfetrat SM, Mehrgardi MA, Aghaei M. Aptamer-conjugated Magnetic Nanoparticles as Targeted Magnetic Resonance Imaging Contrast Agent for Breast Cancer. J Med Signals Sens. 2016;6:243-7. PubMed PMID: 28028501; PubMed Central PMCID: PMC5157001.

11. Mohammadianpanah $M$, Ahmadloo N, Mozaffari MA, Mosleh-Shirazi MA, Omidvari S, Mosalaei A. Primary localized stages I and II non-Hodgkin's lymphoma of the nasopharynx: a retrospective 17year single institutional experience. Ann Hematol. 2009;88:441-7. doi: 10.1007/s00277-008-0627-0. PubMed PMID: 18931844.

12. Mohammadianpanah $M$, Ashouri $Y$, Hoseini $S$, Amadloo N, Talei A, Tahmasebi S, et al. The efficacy and safety of neoadjuvant chemotherapy +/- letrozole in postmenopausal women with locally advanced breast cancer: a randomized phase III clinical trial. Breast Cancer Res Treat. 2012;132:853-61. doi: 10.1007/s10549-0111814-6. PubMed PMID: 22002564.

13. Omidvari S, Shafizad A, Razmjou-Ghalaei S, Nasrolahi $\mathrm{H}$, Ahmadloo N, Ansari M, et al. Efficacy of Topical Honey, Topical Hydrocortisone $1 \%$ and Simple Washing on Healing of Radiation-induced Dermatitis in Breast Cancer Patients. Journal of /sfahan Medical School. 2011;28:1-9.

14. Lawrence S, Seeram E. The current use and effectiveness of bismuth shielding in computed tomography: A systematic review. Radiol Open J. 2017;2:7-16. doi: 10.17140/roj-2-113.

15. Zakariaee SS, Saba V. A Mathematical Head Phantom for Dosimetry Measurements by Monte Carlo Method. Paramedical Sciences and Military Health. 2016;11:12-20.

16. Lambert JW, Gould RG. Evaluation of a Net DoseReducing Organ-Based Tube Current Modulation Technique: Comparison With Standard Dose and Bismuth-Shielded Acquisitions. AJR Am J Roentgenol. 2016;206:1233-40. doi: 10.2214/ AJR.15.15778. PubMed PMID: 27058461.

17. Coursey C, Frush DP, Yoshizumi T, Toncheva G, Nguyen G, Greenberg SB. Pediatric chest MDCT using tube current modulation: effect on radiation dose with breast shielding. AJR Am J Roentgenol. 2008;190:W54-61. doi: 10.2214/AJR.07.2017. PubMed PMID: 18094273.

18. Wang J, Duan X, Christner JA, Leng S, Yu L, McCollough $\mathrm{CH}$. Radiation dose reduction to the breast in thoracic CT: comparison of bismuth shielding, organ-based tube current modulation, and use of a globally decreased tube current. Med Phys. 2011;38:6084-92. doi: 10.1118/1.3651489. PubMed PMID: 22047373.

19. Kim YK, Sung YM, Choi JH, Kim EY, Kim HS. Reduced radiation exposure of the female breast during low-dose chest CT using organ-based tube current modulation and a bismuth shield: comparison of image quality and radiation dose. AJR Am J Roentgenol. 2013;200:537-44. doi: 10.2214/ AJR.12.9237. PubMed PMID: 23436842.

20. Hoang JK, Yoshizumi TT, Choudhury KR, Nguyen GB, Toncheva G, Gafton AR, et al. Organ-based dose current modulation and thyroid shields: techniques of radiation dose reduction for neck CT. AJR Am J Roentgenol. 2012;198:1132-8. doi: 10.2214/ AJR.11.7445. PubMed PMID: 22528904.

21. Lai N, Chen T, Tyan Y, Tsai $H$. Off-centre effect on dose reduction to anterior surfaces with organ-based tube-current modulation. Radiation Measurements. 2013;59:155-9. doi: 10.1016/j.radmeas.2013.04.023.

22. Foley SJ, McEntee MF, Rainford LA. An evaluation of in-plane shields during thoracic CT. Radiat Prot Dosimetry. 2013;155:439-50. doi: 10.1093/rpd/ nct030. PubMed PMID: 23460031.

23. Mathieu KB, Cody DD. Partial arc beam filtration: a novel approach to reducing $\mathrm{CT}$ breast radiation dose. AJR Am J Roentgenol. 2013;200:601-7. doi: 10.2214/AJR.12.8771. PubMed PMID: 23436850; PubMed Central PMCID: PMC3855393.

24. Hopper KD. Orbital, thyroid, and breast superficial radiation shielding for patients undergoing diagnostic CT. Semin Ultrasound CT MR. 2002;23:4237. PubMed PMID: 12509112.

25. Yilmaz MH, Albayram S, Yasar D, Ozer H, Adaletli I, Selcuk D, et al. Female breast radiation exposure during thorax multidetector computed tomography and the effectiveness of bismuth breast shield to reduce breast radiation dose. $J$ Comput Assist Tomogr. 2007;31:138-42. doi: 10.1097/01. rct.0000235070.50055.e6. PubMed PMID: 17259846.

26. Tappouni R, Mathers B. Scan Quality and Entrance Skin Dose in Thoracic CT: A Comparison between Bismuth Breast Shield and Posteriorly Centered Partial CT Scans. ISRN Radiol. 2013;2013:457396. doi: 10.5402/2013/457396. PubMed PMID: 24967274; PubMed Central PMCID: PMC4045517.

27. Hohl C, Wildberger JE, Suss C, Thomas C, Muhlenbruch G, Schmidt T, et al. Radiation dose reduction to breast and thyroid during MDCT: effectiveness of an in-plane bismuth shield. Acta Radiol. 
2006;47:562-7. PubMed PMID: 16875333.

28. Catuzzo P, Aimonetto S, Fanelli G, Marchisio P, Meloni T, Mistretta $L$, et al. Dose reduction in multislice CT by means of bismuth shields: results of in vivo measurements and computed evaluation. Radiol Med. 2010;115:152-69. doi: 10.1007/ s11547-009-0469-4. PubMed PMID: 20012921.
29. Einstein AJ, Elliston CD, Groves DW, Cheng B, Wolff SD, Pearson GD, et al. Effect of bismuth breast shielding on radiation dose and image quality in coronary CT angiography. $J$ Nucl Cardiol. 2012;19:100-8. doi: 10.1007/s12350-011-9473-x. PubMed PMID: 22068687; PubMed Central PMCID: PMC3266996. 